\title{
Comparative Evaluation of Methods Used for Sampling Malaria Vectors in the Kilombero Valley, South Eastern Tanzania
}

\author{
Fredros O. Okumu ${ }^{1,2, *}$, Maya E. Kotas ${ }^{3}$, Japheth Kihonda ${ }^{1}$, Evan Mathenge ${ }^{4}$, Gerry F. Killeen ${ }^{1,5}$ and \\ Sarah J. Moore ${ }^{1,5}$
}

${ }^{1}$ Ifakara Health Institute, Kilombero, Tanzania; ${ }^{2}$ University of Nairobi School of Biological Sciences, Nairobi, Kenya; ${ }^{3}$ Yale University, School of Medicine, New Haven, Connecticut, USA; ${ }^{4}$ Kenya Medical Research Institute, Nairobi, Kenya and ${ }^{5}$ Durham University, School of Biological and Biomedical Sciences, UK

\begin{abstract}
To monitor malaria transmission, effective sampling methods for host seeking vectors are necessary. The suitability of these methods can be determined by field measurements of their trapping efficiencies. We compared the efficiencies of the Human Landing Catch (HLC), the Centers for Disease Control light trap (CDC-LT) placed next to occupied bednets and the Mbita trap for sampling Anopheles gambiae s.l and Anopheles funestus. The sampling methods were rotated through three houses, each with a human bait, for 8 cycles in a $3 \times 3$ Latin-square design. Relative to the HLC, the efficiency (and 95\% c. i) of the CDC-LT for sampling An. gambiae s.l. was 0.331 (0.237-0.460) while that for Mbita trap was 0.031 (0.013 - 0.077). For An. funestus however, the sampling efficiencies were $0.818(0.611-1.096)$ and 0.022 (0.003-0.165) respectively. We conclude that both the CDC-LT placed next to an occupied bednet and the Mbita trap are less efficient than HLC, the latter being evidently unsuitable for use in the Kilombero Valley.
\end{abstract}

Key Words: Anopheles, malaria, sampling methods, Southern Tanzania.

\section{INTRODUCTION}

Adult mosquito sampling methods are essential for monitoring transmission of malaria and other mosquito borne infections. Preference for any sampling method however depends on both its field efficiency and the characteristics of local vector populations. The human landing catch (HLC) method, which involves the use of human bait [1,2], is considered the most reliable technique for sampling adult host seeking mosquitoes. It provides the most accurate measures of man-vector contact, mainly sampling host seeking females, which represent the mosquitoes responsible for disease transmission [3]. Nonetheless there are several drawbacks to the use of HLC [2]. Other than the direct exposure of the catchers to mosquito-borne infections, bias may be introduced by the tiresome and monotonous nature of the method. The method is labour-intensive, expensive and requires close supervision to ensure reliability of the data. There are also variations associated with individual attractiveness $[4,5]$, skill and motivation of the volunteers.

Several attempts have been made to achieve the same useful attributes of HLC with traps which do not necessitate constant human alertness or exposure to disease. Although proxies of human biting rates can be obtained from indoor resting densities [2], these are compromised by increasingly common use of excito-repellent interventions such as insecticide-treated nets and indoor residual spraying $[6,7]$ that force mosquitoes to leave the domestic environment after

*Address correspondence to this author at the Ifakara Health Institute, Kilombero, Tanzania; E-mail: fredros@ihi.or.tz feeding and rest outside [8-10]. Alternatives to human landing catches therefore generally rely on traps baited with humans or with whole human odor. These include Centers for Disease Control light traps (CDC-LT) placed beside occupied untreated bednets [11-13], odor baited entry traps [14] and Mbita trap $[15,16]$. In this category also are the odor baited counter flow geometry traps such as the Mosquito Magnet models of the American biophysics corporation [1719]. All these traps aim at stimulating the host seeking responses of mosquito vectors thus attempting to sample mainly blood seeking females; relevant in disease transmission [3]. Practical alternatives to the controversial HLC however remain elusive.

The CDC-LT, have gained widespread application especially due to their reliability, robustness, ease of use, lower costs of application as compared to HLC and relatively high trapping efficiencies. Various field evaluations have affirmed the effectiveness of the CDC-LT and statistically established correction indices have been proposed to adjust the numbers of mosquitoes sampled [13, 20, 21]. The use of CDC-LT alongside occupied bed nets [22], is being widely adopted as a more sensitive and convenient method for entomological monitoring.

The exposure-free bed net trap, the Mbita trap, was developed on the basis of observations on host seeking mosquitoes around bed nets [16]. The trap is designed to catch the host seeking females without actual contact with the human bait. Field evaluations of the Mbita trap have produced mixed results with some encouraging early results from western Kenya [15, 23] tempered by very low sensitivities reported in other settings $[24,25]$ 
In this study, we sampled mosquitoes from a rural village in south eastern Tanzania using HLC, CDC-LT placed next to occupied untreated bednets and the Mbita trap. Our objective was to determine the sampling efficiencies of the Mbita trap and the CDC-LT placed beside occupied bed nets, relative to HLC, in this ecological setting. Whereas actual species identification would enable other epidemiologically significant estimations, our study focused mainly on (1) the $A n$. gambiae s.l. Giles as a complex and (2) An. funestus Giles. The two groups are also the primary vectors of malaria in the study area [26, 27]. We preferentially paid attention to the comparative evaluations of the different sampling methods; as opposed to the host seeking preferences of species within the An. gambiae and An. funestus complexes. Nonetheless, relevant information on vector species and infection rates for the study area, though not explicitly detailed here, can be inferred from our previous studies, Killeen et al. 2006 [26] Killeen et al. 2007 [28a], and from Charlwood et al. 1995 [28b].

\section{MATERIAL AND METHODS}

\section{Study Area}

This study was conducted in Lupiro Village $\left(8.01^{\circ} \mathrm{S}\right.$ and $\left.36.63^{\circ} \mathrm{E}\right)$, Ulanga District, in the south eastern part of Tanzania. The village lies 300 metres above sea level on the flood plains south of Kilombero Valley and is approximately 26 $\mathrm{km}$ south of Ifakara town. The village experiences very high malaria transmission with recent entomological inoculation rate estimated at 415 infectious bites per year [28a, 29]. The house types are mainly mud and brick walled with thatched roofs but interspersed with a few iron-sheet roofs. The village borders an active perennial swamp extensively cleared for rice cultivation. This, apart from being the economic mainstay of a considerable proportion of the population, is a massive mosquito breeding site and arguably a further predisposition to malaria infections [30]. Annual rainfall is approximately $1200-1800 \mathrm{~mm}$ and the village has an annual temperature range of between $20^{\circ} \mathrm{C}$ and $32.6^{\circ} \mathrm{C}$.

\section{Mosquito Collections}

Sampling was conducted nightly in a $3 \times 3$ Latin square design replicated 8 times in the period between $18^{\text {th }}$ July and $10^{\text {th }}$ August 2004. Three sampling methods: HLC, Mbita Trap and CDC-LT (John W. Hock Co. USA) set near an occupied untreated bed net were tested. Three volunteers who were recruited on written consent and trained on the different sampling methods participated in the study. The 3 volunteers were allocated to 3 houses, of approximately similar dimensions and design, and sampling methods were rotated nightly, so that each individual was coupled with each sampling method 8 times over the course of the trial. Thus on each experimental night one of the volunteers slept under Mbita trap, another under an untreated bed net next to a CDC-LT and the third volunteer performed HLC. The three houses were located approximately 200 meters apart. To combine the variations associated with houses and individual volunteers, we fixed the volunteer-house pairs (hereafter referred to simply as "location") so that each volunteer remained in the same house for the entire experimental period. The fixed pairing was additionally convenient since the selected houses actually belonged to the respective volun- teers. The human landing catches were conducted indoors for a period of 45 minutes every hour, the volunteer taking 15 minutes for snacks and rest each time. All the experiments ran between $1800 \mathrm{Hrs}$ and $0600 \mathrm{Hrs}$, during which only the participating volunteers stayed in the experimental houses.

The CDC light traps were set with the opening approximately 1 meter above the floor and as close as possible to the feet the bednet occupant; as previously described by Mboera et al. 1998 [11]. A wet cotton wool pad was put into the small pouch inside the CDC light trap receptor to prevent desiccation of captured mosquitoes. On the other hand, the Mbita trap was hung over the bed so that the occupant was wholly covered. The entry cone-end of the trap was kept approximately at the eaves level. The volunteer occupant slept under the trap during the experimental period at the end of which the entry hole of the trap was plugged with cotton wool.

\section{Processing of Samples}

Every morning samples were collected and brought to the field processing table. Live mosquitoes were anaesthetized and killed in a modified killing bottle using $70 \%$ ethanol. The mosquitoes were sorted into species and counted. Morphological classification was used to group the adults as $A n$. gambiae s.l., An. funestus or culicines [31]. Any other species found; which infrequently included other non-malaria anophelines were disregarded.

\section{Protection of Participants}

Following a thorough explanation of the risks involved and the objectives of the study, written informed consents were obtained from all the volunteers. All participants were provided with immediate access to weekly screening for malaria parasites by light microscopy and treatment with artemether-lumefantrine. Ethical review and approval of the study was provided by the Medical Research Coordination Committee of the National Institute for Medical Research of the United Republic of Tanzania (Reference numbers NIMR/ HQ/R8a/VolVIII/1, NIMR/HQ/R.8a/VOL.IX/324 and NIMR/ HQ/R.8a/VOL.X/12).

\section{Data Analysis}

Data from the HLC was divided by 0.75 , i.e. $45 / 60$ to account for the fact that HLC had been performed for only 45 minutes of each hour, whereas CDC-LT and Mbita traps were run continuously. Data was fitted to Generalized Linear Models (GLMs) using SPSS 15 (SPSS Inc, Chicago) as follows: Mosquito catches were modeled as a function of sampling method and location; treating location as the experimental unit and HLC as the reference method. We treated date as a random factor to account for fluctuations in daily mosquito numbers and any resultant density dependent effect on trap efficiencies. Due to skewness, the mosquito counts were log-linked in the generalized estimating equations to make the data amenable to assumptions of the standard normal distribution. The reported trap efficiencies were therefore estimated by exponentiating the best fit model parameters for each trap type. We also performed Tukey's Honestly Significant Difference test to measure the significance between the means of mosquitoes collected using each sampling method and each location. 


\section{RESULTS}

In the 24 trap nights, 4913 mosquitoes were collected, comprising 4436 An. gambiae s.l., 104 An. funestus and 373 culicine mosquitoes. Of all the mosquitoes, $3583(72.93 \%)$ were caught by the HLC, $1182(24.06 \%)$ in the CDC-LT and $148(3.01 \%)$ in the Mbita Trap. A summary of the mean adult mosquito collections per trap per species is given in Table 1.

Based on the HLC as the reference method, the efficiencies (and 95\% c. i.) of the CDC-LT and the Mbita Trap for sampling An. gambiae s.l. were 0.331 (0.237 - 0.460), $\mathrm{P}<$ 0.001 and $0.031(0.013-0.077), \mathrm{P}<0.001$ respectively. On the other hand the CDC-LT had a sampling efficiency of 0.818 (.0.611 - 1.096), $\mathrm{P}=0.179$ for An. funestus while the Mbita trap efficiency was $0.022(0.003-0.165), \mathrm{P}<0.001$ for the same species. When data for all culicine species was pooled, the efficiencies of the CDC-LT and the Mbita trap were $0.578(0.362-0.923), \mathrm{P}=0.022$ and 0.031 (0.021$0.046) \mathrm{P}<0.001$ respectively. In all cases, greater than $70 \%$ of the culicines trapped were Culex quinquefasciatus species.

Catches of An. gambiae at any location were significantly dependent on sampling method $(\mathrm{P}<0.001, \mathrm{~F}=152.06, \mathrm{df}=$ $2)$ and location $(\mathrm{P}<0.001, \mathrm{~F}=53.36$, df $=2)$. The interaction between sampling method and location also significantly affected the number of An. gambiae s.l. collected ( $\mathrm{P}<$ $0.001, \mathrm{~F}=8.63 \mathrm{df}=4$ ). No significant difference tests were conducted on An. funestus data separately due to the very low catches of this species. Whereas sampling method significantly affected the number of culicines caught at any location $(\mathrm{P}<0.001, \mathrm{~F}=35.03$, df $=2)$, we found no significant effect of either location $(\mathrm{P}=0.53, \mathrm{~F}=0.67, \mathrm{df}=2)$ or the interaction between sampling method and location $(\mathrm{P}=$ $0.58, \mathrm{~F}=0.73, \mathrm{df}=4)$. For all the mosquito species however, there was also a significant influence of day on the mosquito catches $(\mathrm{P}=0.001,3.20, \mathrm{~F}=23)$.

\section{DISCUSSION}

The suitability of mosquito sampling methods may vary alongside geographic and ecological differences, necessitating the calibration of sampling tools for use in different settings. Our study was an effort to calibrate the recently devel- oped Mbita trap and the CDC-LT for use within the Kilombero Valley, south eastern Tanzania. We have determined that the HLC is more efficient than both the CDC-LT and the Mbita trap in the study area. Though these findings support previous arguments that the HLC is the most appropriate sampling method for anthropophilic Anopheles mosquitoes $[2,12,23,32,33]$, the computed sampling efficiencies are of substantial epidemiological significance. However, to avoid overestimation or underestimation of malaria transmission, case by case calibration of CDC-LT to HLC $[12,13,34]$ is recommended. Moreover in this setting though the CDC-LT catches only $33 \%$ as many An. gambiae as the HLC, this is amply sufficient for evaluations of malaria transmission intensity at the vector densities established in Kilombero Valley $[26,28]$.

The Mbita trap performance was far poorer than that of the CDC-LT. Over the entire sampling period the trap caught only 140 anophelines (137 An. gambiae s.l. and 3 An. funestus) comprising a mere $3.08 \%$ of the total anophelines caught by the three methods. Since its development [16], only a few field evaluations have been conducted on the Mbita trap, but like Braimah et al. 2005 [25] and Laganier et al. 2003 [24], the trap efficiencies we report here are much lower than those reported from the semi field and field studies in western Kenya [15, 23]. Comparison of our results with previous studies is shown in Table $\mathbf{2}$. Whereas the very low mosquito counts limited our assessment of relationships between the Mbita trap and the other two methods, they evidently illustrate inneficiency of the trap. Laganier et al. 2003 [24] computed a remarkably low sampling efficiency of 0.06 for An. gambiae s.l. with the Mbita trap. One important postulation by this research group was that the underperformance of the trap could have been as a result of the exophilic and exophagic preferences of the anopheles mosquitoes in Madagascar [24]. Here we report similarly low efficiencies in an area of predominantly endophagic and endophilic $A n$. gambiae s.l. and An. funestus vectors [26]. As such, the feeding and resting behavior of mosquitoes appear not to be the true limiting determinant of the Mbita trap performance. Thus there is still a necessity to focus research towards development of a reliable, exposure-free and affordable battery less-replacement for the CDC-LT, a need which the Mbita trap was intended to fulfill.

Table 1. Comparison of Mosquito Counts Collected by Different Sampling Methods

\begin{tabular}{|c|c|c|c|c|c|c|}
\hline \multirow{2}{*}{$\begin{array}{l}\text { Sampling } \\
\text { Method }\end{array}$} & \multicolumn{2}{|c|}{ An. gambiae s.l. } & \multicolumn{2}{|c|}{ An. funestus } & \multicolumn{2}{|c|}{ Culicines } \\
\hline & $\begin{array}{c}\text { Mean } \\
(95 \% \mathrm{CI})\end{array}$ & $\begin{array}{c}\text { Estimated Efficiency } \\
(95 \% \mathrm{CI})\end{array}$ & $\begin{array}{c}\text { Mean } \\
(95 \% \mathrm{CI})\end{array}$ & $\begin{array}{c}\text { Estimated Efficiency } \\
(95 \% \text { CI })\end{array}$ & $\begin{array}{c}\text { Mean } \\
(95 \% \mathrm{CI})\end{array}$ & $\begin{array}{c}\text { Estimated Efficiency } \\
(95 \% \text { CI })\end{array}$ \\
\hline HLC & $\begin{array}{c}143.24 \\
(89.03-197.44) \mathrm{a}\end{array}$ & Reference & $\begin{array}{c}2.60 \\
(1.55-3.65) \mathrm{a}\end{array}$ & Reference & $\begin{array}{c}9.95 \\
(4.24-15.65) \mathrm{a}\end{array}$ & Reference \\
\hline Mbita Trap & $\begin{array}{c}5.71 \\
(2.97-8.45) \mathrm{c}\end{array}$ & $\begin{array}{c}0.031 \\
(0.01-0.08)\end{array}$ & $\begin{array}{c}0.13 \\
(-0.01-0.26) \mathrm{c}\end{array}$ & $\begin{array}{c}0.022 \\
(0-0.17)\end{array}$ & $\begin{array}{c}0.33 \\
(0-0.67) \mathrm{c}\end{array}$ & $\begin{array}{c}0.031 \\
(0.02-0.05)\end{array}$ \\
\hline
\end{tabular}

Differing letters a, b, c indicate significance difference between means of the different sampling methods for each species (P < 0.05). Sampling efficiencies were estimated based on the HLC data as the reference. 
Table 2. Comparison of Results from Different Evaluations of the Mbita Trap

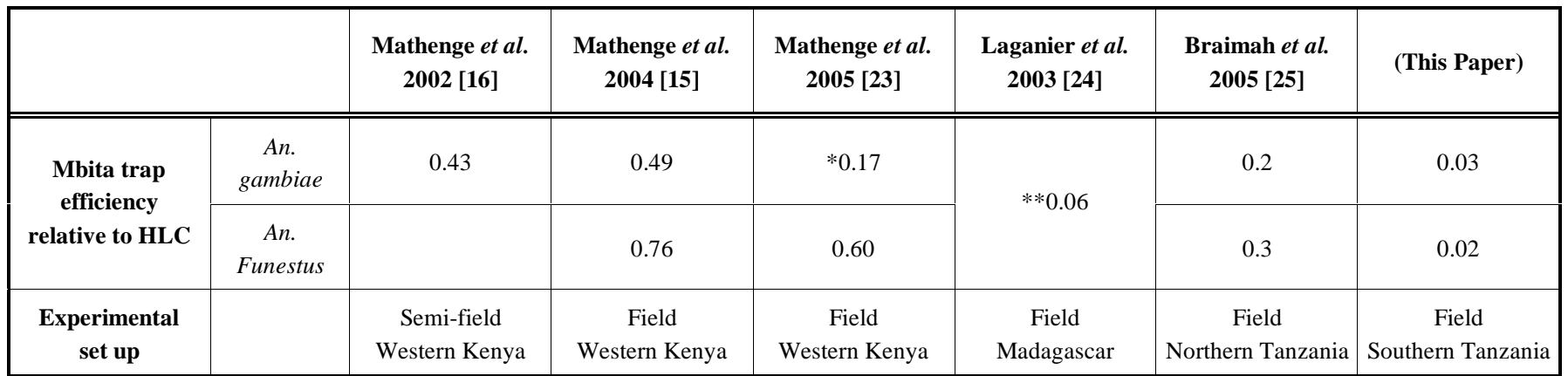

*Sampling efficiency calculated for An. arabiensis

** Data lumped for all anophelines.

\section{CONCLUSION}

Our evaluation clearly indicate that the efficiencies of both the CDC-LT placed beside an occupied bednet and the Mbita trap are lower than the efficiency of the HLC but the former is nevertheless suitable for measuring representative human-biting rates. Due to its exceedingly low efficiency reported both in our study and in the previous studies, we are convinced that the Mbita trap is not suitable and therefore should not be used for sampling mosquito populations in the Kilombero Valley or in other comparable ecological settings. Consequently, there remains a need for new sampling tools that offer the envisaged advantages of the Mbita trap but that would effectively sample the malaria vector populations. We recommend that practical application of our results be limited to similar ecological settings. Furthermore, similar calibration measurements should be conducted on a case-by-case basis prior to introduction of new sampling methods in ecologically distinct areas.

\section{AUTHORS' CONTRIBUTIONS}

FOO, MEK and JK conducted and supervised the field experiments in consultation with EM and GFK. FOO and SJM wrote the first draft of the manuscript and performed the statistical analysis of the data. GFK conceived, supervised and obtained funding for the study. GFK and EM helped edit the manuscript into its final form.

\section{ACKNOWLEDGEMENTS}

We thank the community volunteers for participating in the experiments and the residents of Lupiro Village for the hosting us during our time working in the village. This project was funded by the National Institutes of Health of the Unites States of America.

\section{REFERENCES}

[1] WHO. Manual on practical entomology in malaria. Part II: Methods and techniques. Geneva; 1975.

[2] Service MW. A critical review of procedures for sampling populations of adult mosquitoes. Bull Entomol Res 1977; 67: 343-82.

[3] MacDonald G. Epidemiological basis of malaria control. Bull WHO 1956; 15: 613-26.

[4] Takken W, Knols BG. Odor-mediated behavior of Afrotropical malaria mosquitoes. Annu Rev Entomol 1999; 44: 131-57.

[5] Mukabana WR, Takken W, Knols BGJ. Host-specific cues cause differential attractiveness of Kenyan men to the African malaria vector Anopheles gambiae. Malar J 2002; 1: 17.
[6] WHO. World malaria report 2005. Geneva: 2005.

[7] WHO. Roll back malaria global strategic plan 2005-2015. Geneva: 2005.

[8] Muirhead-Thomson RC. The significance of irritability, behaviouristic avoidance and allied phenomena in malaria eradication. Bull WHO 1960; 22: 721-34.

[9] Magesa SM, Wilkes TJ, Mnzava AEP, et al. Trial of pyrethroid impregnated bednets in an area of Tanzania holoendemic for malaria. Part 2: Effects on the malaria vector population. Acta Trop 1991; 49: 97-108.

[10] Bøgh C, Pedersen EM, Mukoko DA, et al. Permethrin-impregnated bed net effects on resting and feeding behaviour of lymphatic filariasis vector mosquitoes in Kenya. Med Vet Entomol 1998; 12: 52-9.

[11] Mboera LE, Kihonda J, Braks MA, et al. Short report: Influence of centers for disease control light trap position, relative to a humanbaited bed net, on catches of Anopheles gambiae and Culex quinquefasciatus in Tanzania. Am J Trop Med Hyg 1998; 59: 595-6.

[12] Hii JLK, Smith T, Mai A, et al. Comparison between anopheline mosquitoes (Diptera: Culicidae) caught using different methods in a malaria endemic area of Papua New Guinea. Bull Entomol Res 2000; 90: 211-9.

[13] Davis JR, Hall T, Chee EM, et al. Comparison of sampling anopheline mosquitoes by light-trap and human-bait collections indoors at Bagamoyo, Tanzania. Med Vet Entomol 1995; 9: 249-55.

[14] Constantini C, Gibson G, Brady J, et al. A new odor baited traps to collect host seeking mosquitoes. Parassitologia 1993; 35: 5-9.

[15] Mathenge EM, Omweri GO, Irungu LW, et al. Comparative field evaluation of the Mbita trap, the centers for disease control light trap, and the human landing catch for sampling of malaria vectors in western Kenya. Am J Trop Med Hyg 2004; 70: 33-7.

[16] Mathenge E, Killeen GF, Oulo D, et al. Development of an exposure-free bednet trap for sampling Afrotropical malaria vectors. Med Vet Entomol 2002; $16: 67-74$

[17] Njiru BN, Mukabana RW, Takken W, et al. Trapping of the malaria vector Anopheles gambae with odour baited MM-X traps in semi field conditions in western Kenya. Malar J 2006; 5: 39.

[18] Kline DL. Traps and trapping techniques for adult mosquito control. J Am Mosq Control Assoc 2006; 23: 490-6.

[19] Kline DL. Comparison of two American biophysics mosquito traps: The professional and the new counterflow geometry trap. J Am Mosq Control Assoc 1999; 15: 276-82.

[20] Shiff CJ, Minjas JN, Hall T, et al. Malaria infection potential of anopheline mosquitoes sampled by light trapping indoors in coastal Tanzanian villages. Med Vet Entomol 1995; 9: 256-62.

[21] Lines JD, Curtis CF, Wilkes TJ, et al. Monitoring human-biting mosquitoes (Diptera: Culicidae) in Tanzania with light-traps hung beside mosquito nets. Bull Entomol Res 1991; 81: 77-84.

[22] Garrett-Jones C, Magayuka SA. Studies on the natural incidence of Plasmodium and Wuchereria infections in anopheles in rural East Africa: 1-Assessment of densities by trapping hungry female Anopheles gambiae Giles Species. WHO/Mal/75851. Geneva: WHO 1975.

[23] Mathenge E, Misiani, Oulo DO, et al. Comparative performance of the Mbita trap, CDC light trap and the human landing catch in the sampling of Anopheles arabiensis, An. funestus and culicine spe- 
cies in a rice irrigation scheme in western Kenya. Malar J 2005; 4: 7.

[24] Laganier R, Randimby FM, Rajaonarivelo V, et al. Is the Mbita trap a reliable tool for evaluating the density of anopheline vectors in the highlands of Madagascar? Malar J 2003; 2: 42.

[25] Braimah N, Drakely C, Kweka E, et al. Tests of bednet traps (Mbita traps) for monitoring mosquito populations and time of biting in Tanzania and possible impact of prolonged ITN use. Int $\mathbf{J}$ Trop Insec Sci 2005; 00: 1-6.

[26] Killeen GF, Kihonda J, Lyimo E, et al. Quantifying behavioural interactions between humans and mosquitoes: Evaluating the insecticidal efficacy of insecticidal nets agains malaria transmission in rural Tanzania. BMC Infec Dis 2006; 6: 161.

[27] Drakeley C, Schellenberg D, Kihonda J, et al. An estimation of the entomological inoculation rate for Ifakara: A semi-urban area in a region of intense malaria transmission in Tanzania. Trop Med Int Health 2003; 8: 767-74.

[28] (a) Killeen GF, Tami A, Kihonda J, et al. Cost-sharing strategies combining targeted public subsidies with private-sector delivery achieve high bednet coverage and reduced malaria transmission in Kilombero Valley, southern Tanzania. BMC Infec Dis 2007; 71: 21; (b) Charlwood JD, Smith T, Kihonda J, et al. Density inde- pendent feeding success of malaria vectors (Diptera: Culicidae) in Tanzania. Bull Entomol Res 1995; 85: 29-35.

[29] Killeen GF, Kihonda J, Lyimo E, et al. Quantifying behavioural interactions between humans and mosquitoes: Evaluating the insecticidal efficacy of insecticidal nets agains malaria transmission in rural Tanzania. BMC Infec Dis 2006; 6: 161.

[30] Ijumba J, Lindsay S. Impact of irrigation on malaria in Africa: Paddies paradox. Med Vet Entomol 2001; 15: 1-11.

[31] Gillies MT, Coetzee M. A supplement to the Anophelinae of Africa South of the Sahara (Afrotropical region). Johannesburg: South African Medical Research Institute 1987.

[32] Sithiprasasna R, Jaichapor B, Chanaimongkol S, et al. Evaluation of candidate traps as tools for conducting surveillance for Anopheles mosquitoes in a malaria endemic area in Western Thailand. J Med Entomol 2004; 41: 151-7.

[33] Dia I, Diallo D, Duchemin JB, et al. Comparisons of human landing catches and odor baited entry for sampling malaria vectors in Senegal. J Med Entomol 2005; 42: 104-9.

[34] Mbogo CN, Glass GE, Forster D, et al. Evaluation of light traps for sampling anopheline mosquitoes in Kilifi, Kenya. J Am Mosq Control Assoc 1993; 9: 260-3.

(c) Okumu et al.; Licensee Bentham Open.

This is an open access article licensed under the terms of the Creative Commons Attribution Non-Commercial License (http://creativecommons.org/licenses/ by-nc/3.0/) which permits unrestricted, non-commercial use, distribution and reproduction in any medium, provided the work is properly cited. 